ARTICLE

Received 14 May 2013 | Accepted 2 Aug 2013 | Published 3 Sep $2013 \quad$ DOl: 10.1038/ncomms3392

\title{
Role of RIM1 $\alpha$ in short- and long-term synaptic plasticity at cerebellar parallel fibres
}

\author{
Michael Kintscher ${ }^{1}$, Christian Wozny ${ }^{1}$, Friedrich W. Johenning ${ }^{1}$, Dietmar Schmitz ${ }^{1,2,3,4, \star} \&$ Jörg Breustedt ${ }^{1, \star}$
}

The presynaptic terminals of synaptic connections are composed of a complex network of interacting proteins that collectively ensure proper synaptic transmission and plasticity characteristics. The key components of this network are the members of the RIM protein family. Here we show that RIM1 $\alpha$ can influence short-term plasticity at cerebellar parallelfibre synapses. We demonstrate that the loss of a single RIM isoform, RIM1 $\alpha$, leads to reduced calcium influx in cerebellar granule cell terminals, decreased release probability and consequently an enhanced short-term facilitation. In contrast, we find that presynaptic longterm plasticity is fully intact in the absence of RIM1 $\alpha$, arguing against its necessary role in the expression of this important process. Our data argue for a universal role of RIM1 $\alpha$ in setting release probability via interaction with voltage-dependent calcium channels at different connections instead of synapse-specific functions.

\footnotetext{
${ }^{1}$ Neuroscience Research Center, Charité-Universitätsmedizin Berlin, Charitéplatz 1, 10117 Berlin, Germany. ${ }^{2}$ Cluster of Excellence Neurocure, CharitéUniversitätsmedizin Berlin, Charitéplatz 1, 10117 Berlin, Germany. ${ }^{3}$ Bernstein Center for Computational Neuroscience Berlin, 10115 Berlin, Germany.

${ }^{4}$ Deutsches Zentrum für Neurodegenerative Erkrankungen in der Helmhotz-Gemeinschaft, 10115 Berlin, Germany. ${ }^{\star}$ These authors contributed equally to this work. Correspondence and requests for materials should be addressed to J.B. (email: joerg.breustedt@charite.de).
} 
$\mathrm{T}$ he plasticity of synaptic connections is a core feature of neuronal networks that can be divided into short-term plasticity (STP, ms to s) and long-term plasticity (LTP, min to $\mathrm{h}$ ) depending on duration. The molecular composition of synapses is critically important in determining STP and LTP, and generally the underlying cellular and molecular mechanisms are different.

STP acts on synaptic connections between neurons on a fast time scale and the underlying events are for the most part taking place on the presynaptic side of a connection ${ }^{1}$. The direction of STP at a given synapse-that is, depression or facilitationdepends mostly on the initial release probability $\left(P_{\mathrm{r}}\right)$ of a given synapse $^{2}$. Among the factors that influence the $P_{\mathrm{r}}$ are: the number of vesicles in the releasable pool, the number and type of voltagedependent calcium channels (VDCCs), the spacing between the VDCCs and synaptic vesicles as well as the specific proteins mediating the priming and calcium sensitivity of release ${ }^{3}$.

For the majority of synapses, LTP is both induced as well as expressed on the postsynaptic side ${ }^{4}$, ultimately leading to an alteration in AMPA receptor number or function. In contrast, only a rather specialized set of synapses displays presynaptic induction and expression of LTP, in which the synapses are finally strengthened through an increase in presynaptic transmitter release most likely involving changes at the release machinery ${ }^{5}$. Examples for this presynaptic type of LTP can be found in hippocampal mossy fibres ${ }^{6}$, cerebellar parallel fibres $(\mathrm{PF})^{7}$ and corticolateral amygdala synapses $^{8}$ as well as the subiculum ${ }^{9}$.

Rab3-interacting molecule (RIM) $1 \alpha$ is a presynaptically expressed protein of the active zone that has a key role in the docking and priming of synaptic vesicles ${ }^{10}$. It has also been implicated to be of great importance in both presynaptic STP and LTP. Remarkably, RIM1 $\alpha$ was shown to be essential for LTP but not for STP at some connections (hippocampal mossy fibre and cerebellar PF synapses ${ }^{11}$, as well as hippocampal GABAergic synapses $^{12}$ ). In contrast, it was necessary for STP but not for LTP at the hippocampal Schaffer-collateral synapses ${ }^{13}$. The RIM interaction partner Rab3 displays a similar phenotype in that it influences STP at the hippocampal pyramidal neurons ${ }^{14,15}$ and LTP, but not STP, at the mossy fibre synapses ${ }^{16}$. Therefore, synapses that show presynaptic LTP seem to rely on RIM1 $\alpha$ for the expression, but STP at these connections remains unaffected by RIM1 $\alpha$ loss. On the other hand, synaptic connections that obviously do not need RIM1 $\alpha$ for LTP expression because of its postsynaptic origin do show altered STP without RIM1 $\alpha$. The only exception to this scheme described so far is the corticoamygdala synapse where both presynaptic LTP as well as STP are affected by RIM1 $\alpha \operatorname{loss}^{17}$.

More recently, the detailed mechanism how RIM operates was elegantly demonstrated at the calyx of Held ${ }^{18}$ and in hippocampal cell cultures ${ }^{19,20}$ using RIM1/2-conditional knockout (KO) mice. Those studies demonstrated that RIM assists in targeting calcium channels to active zones. RIM deletion resulted in a reduction in calcium current density at the calyx as well as a reduction in action potential-driven calcium transients in presynaptic boutons of the hippocampal pyramidal neurons. RIM was also shown to be important for the establishment of the size of the readily releasable pool and priming of synaptic vesicles. As a consequence of all the aforementioned effects, the $P_{\mathrm{r}}$ is reduced in the absence of RIM, albeit only slightly, nevertheless leading to reduced short-term depression at the calyx of Held.

For presynaptic LTP, the general consensus holds that a rise in presynaptic terminal calcium is necessary for induction ${ }^{5,21,22}$. Therefore, a subsequent question is, considering the interaction of RIM with VDCCs, whether the loss of RIM1 $\alpha$ may lead to a failure of presynaptic LTP induction rather than having a role in LTP expression.
In this report, we show that the deletion of a single RIM isoform, RIM1 $\alpha$, already causes a detectable phenotype at the cerebellar PF synapses in the form of reduced calcium influx leading to a reduced $P_{\mathrm{r}}$ and consequentially an increased shortterm facilitation. Moreover, we demonstrate here that despite the absence of RIM1 $\alpha$, long-term potentiation can be normally induced and expressed. Our data argue therefore for a universal role of RIM1 $\alpha$ in setting the $P_{\mathrm{r}}$ of synapses but do not support an indispensable function for presynaptic LTP expression.

\section{Results}

Enhanced short-term facilitation. For this study we chose to investigate the cerebellar PF synapse-in this particular case meaning the connection of granule cells on Purkinje neurons. In a first set of experiments, we determined the properties of STP in the absence of RIM1 $\alpha$ by recording from slices from genetic deletion mice. All numerical data are provided as \pm s.e.m. We found a significant enhancement of paired-pulse facilitation of excitatory postsynaptic potentials (EPSCs) recorded from Purkinje neurons in RIM1 $\alpha(-/-)$ mice compared with the control (Fig. 1a, PPR for 50-ms ISI, WT: $1.76 \pm 0.05, n=14$ cells, four mice versus KO $1.92 \pm 0.05, n=16$ cells; six mice; $P=0.036$, Student's $t$-test). We next applied a train of stimuli, consisting of 25 pulses delivered at $14 \mathrm{~Hz}$, and also observed a considerably greater response over the whole time course of the train in KO mice (Fig. 1b; average of twenty first to twenty fifth pulses, WT: $1.4 \pm 0.09, n=10$ cells, four mice versus KO: $1.9 \pm 0.1, n=11$ cells, three mice; $P=0.002$, Student's $t$-test, values normalized with respect to the first pulse in the train). These data demonstrate an enhanced short-term facilitation in the absence of RIM1 $\alpha$.

Reduced $\boldsymbol{P}_{\mathbf{r}}$. We then wanted to test whether the increased shortterm facilitation might be caused by an alteration in $P_{\mathrm{r}}$ because of the loss of RIM1 $\alpha$. For this purpose we performed two sets of experiments. In the first series, we made use of the competitive low affinity AMPA receptor antagonist $\gamma$-D-glutamylglycine $(\gamma D G G)$ $(1 \mathrm{mM})$. We indeed found a stronger reduction in the amplitude of EPSCs of the first as well as second pulses in recordings from knockout compared with wild-type mice (Fig. 2, first pulse WT: $0.45 \pm 0.02$ versus KO: $0.38 \pm 002, P=0.008$ and second pulse WT: $0.59 \pm 0.03$ versus KO: $0.46 \pm 0.021 ; P=0.003$, Student's $t$-test, $n=7$ cells and three mice each). Our data are in line with the assumption that the absence of RIM1 $\alpha$ leads to a reduction in $P_{\mathrm{r}}$, which in turn is reflected by a smaller glutamate transient in the synaptic cleft and therefore reduced displacement of $\gamma$ DGG from its receptors. As a control experiment, subsequent application of low concentrations of NBQX $(200 \mathrm{nM})$ in contrast displayed similar depression of both EPSC responses in a paired-pulse stimulation protocol (Fig. 2c, PPR wash $=2.1 \pm 0.06$ versus PPR in $\mathrm{NBQX}=2.2 \pm 0.09$, not significant, one-way analysis of variance (ANOVA) with Bonferroni's multiple comparison, $n=5$ ).

Next, we performed a variance-mean analysis. Here EPSCs were recorded at five different external calcium concentrations and the variance was calculated from at least 30 samples at each epoch and plotted against the mean amplitude of the respective EPSC (Fig. 3a-c). We detected a significantly reduced $P_{\mathrm{r}}$ in RIM1 $\alpha-/-$ mice at concentrations from 2.5 to $4.5 \mathrm{mM}$ calcium in comparison to responses from wild-type mice (Fig. $3 \mathrm{~d}, 2.5 \mathrm{mM}$ $\mathrm{Ca}^{2}+P_{\mathrm{r}}$ WT: $0.28 \pm 0.03, n=5$ cells per four mice versus $\mathrm{KO}$ $0.14 \pm 0.03, n=5$ cells per three mice; $P=0.009$ and $4.5 \mathrm{mM}$ $\mathrm{Ca}^{2+}$ WT: $0.66 \pm 0.04$ versus KO $0.45 \pm 0.05 ; P=0.011$, Student's $t$-test). Both at the low $(1 \mathrm{mM})$ and high end $(5.5 \mathrm{mM})$ of concentrations tested (Fig. $3 \mathrm{~d}, 5.5 \mathrm{mM} \mathrm{Ca}^{2+}, P_{\mathrm{r}}$ WT: $0.84 \pm 0.05$ versus KO $0.77 \pm 0.05 ; P=0.36$, Student's $t$-test) no difference in $P_{\mathrm{r}}$ was discernible between the two genotypes. The latter indicates 


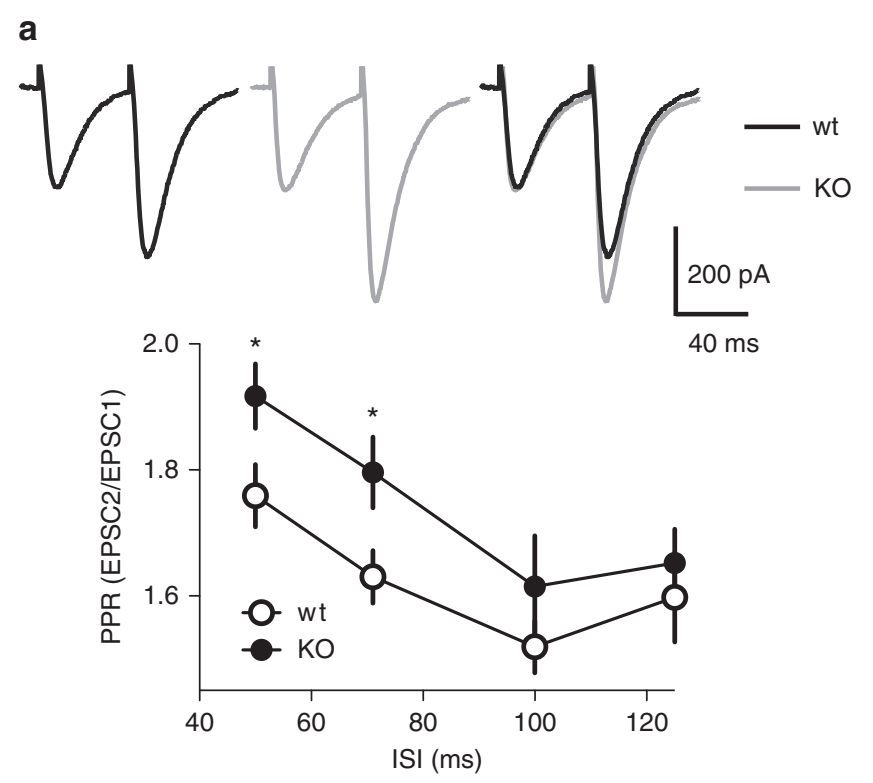

b
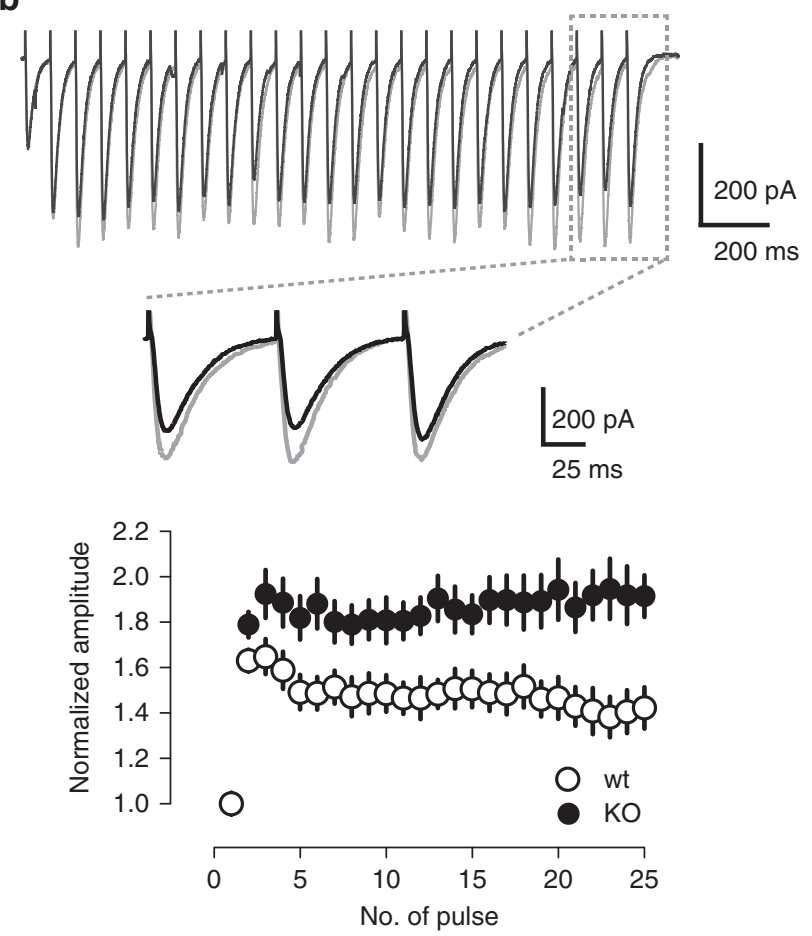

Figure 1 | Increased short-term plasticity in RIM1 $\alpha$ knockout mice.

Short-term facilitation was assessed by paired-pulse and train stimulation. (a) Paired pulses were applied with an interstimulus interval (ISI) of 50, 71, 100 and $125 \mathrm{~ms}$. Representative traces (top) are shown for an ISI of $50 \mathrm{~ms}$. Summary graph (bottom) for all ISIs, wild type (open circles) and knockout (filled circles); ISI $50 \mathrm{~ms}: \mathrm{n}(\mathrm{WT})=14, \mathrm{n}(\mathrm{KO})=16$; ISI $71 \mathrm{~ms}: \mathrm{n}(\mathrm{WT})=10$, $\mathrm{n}(\mathrm{KO})=11$; data points denote mean \pm s.e.m. ${ }^{\star} P<0.05$, Student's t-test. (b) A $14-\mathrm{Hz}$ train was applied for 25 pulses. Example traces (top) and summary plot (bottom) are shown; $\mathrm{n}(\mathrm{WT})=10, \mathrm{n}(\mathrm{KO})=11$, data report mean \pm s.e.m. The first two train pulses in (b) correspond to the 71-ms ISI in (a). The inset shows the last three responses in the train on an expanded time scale.

that the release defect introduced by the deletion of RIM $1 \alpha$ can be overcome by raising external calcium. In contrast, within a moderate range of calcium concentrations, deletion of RIM $1 \alpha$ leads to a reduction in $P_{\mathrm{r}}$. The averaged quantal amplitude-that is, the response size to a released quantum-was similar in wildtype and RIM1 $\alpha-/-$ mice (Fig. $3 \mathrm{e}, Q_{\mathrm{av}}$ WT: $3.5 \pm 0.16 \mathrm{pA}$ versus KO: $3.9 \pm 0.36 \mathrm{pA}$ ).

Reduced calcium influx in presynaptic boutons. The obvious next question was by which mechanism RIM1 $\alpha$ might affect $\mathrm{P}_{\mathrm{r}}$ ? Is it a direct function of RIM $1 \alpha$ at the release machinery or rather an indirect process via interaction with VDCCs?

To address this issue, we investigated whether RIM1 $\alpha$ deletion affects calcium influx in the presynaptic granule cell axon terminals. Granule cells were patched and filled with a combination of two dyes, Alexa-594 (red channel) and Fluo4FF (green channel). At least $20 \mathrm{~min}$ after establishment of the wholecell configuration lines, scans through individual granule cell boutons were recorded and both calcium-insensitive red signals as well as calcium responses (green signal) were recorded in epiand transfluorescence pathways (Fig. 4). In order to calculate the action potential-driven calcium influx into the terminals, we divided the green by the red signal and estimated the calcium concentration change according to established methods (refs 23,24 see methods). We performed two sets of experiments; on one hand looking at boutons from the ascending segment (AA) of the granule cell axon and at the PF segment on the other. Those two segments have been described to have physiologically different properties and therefore should be distinguished ${ }^{25,26}$. Notably, the calculated peak calcium-influx change was considerably smaller in the terminals from RIM $1 \alpha(-/-)$ than in wild-type mice both in boutons from the ascending axon and from the PF segment (Fig. 4d,e, for PF: WT $=0.31 \pm 0.02 \mu \mathrm{M}$, $n=38$ boutons per three mice versus $\mathrm{KO}=0.19 \pm 0.01 \mu \mathrm{M}$, $n=35$ boutons per three mice; $P<0.001$, and for AA: $\mathrm{WT}=0.42 \pm 0.03 \mu \mathrm{M}, n=23$ boutons per eight mice versus $\mathrm{KO}=0.31 \pm 0.02 \mu \mathrm{M}, n=23$ boutons per six mice, $P=0.013$, Student's $t$-test). These data indicate a profound contribution of the RIM1 $\alpha$ isoform to VDCC function or trafficking in presynaptic boutons of cerebellar granule cells. This reduction in calcium influx is most likely causal for the remarkable reduction in underlying $P_{\mathrm{r}}$ and is reflected in altered STP behaviour in the absence of RIM1 $\alpha$.

Intact expression of long-term potentiation. We finally wanted to explore the possible consequences that the reduced calcium influx in the absence of RIM1 $\alpha$ might have on the induction and expression of presynaptic LTP at parallel-fibre synapses. The necessity of a rise in presynaptic terminal calcium for the induction of presynaptic LTP is well established ${ }^{5}$, and it is therefore conceivable that the observed reduction in calcium entry might lead to a failure of LTP induction. To begin with, we induced chemical LTP by application of $50 \mu \mathrm{M}$ forskolin. In slices from both WT as well as RIM $1 \alpha-/-$, LTP was readily induced and expressed and showed no differences between the two genotypes (Fig. 5a, WT 162.4 $\pm 11.3 \%, n=9$ slices, five mice versus $\mathrm{KO} 165.5 \pm 10.6 \%, n=9$ slices, seven mice; $P=0.84$, Student's $t$-test, values taken from averages of $26-30 \mathrm{~min}$ after induction). In the same lines, LTP induced by tetanic stimulation ( $8 \mathrm{~Hz}$ for $25 \mathrm{~s}$ ) was also of the same magnitude in RIM1 $\alpha-/-$ as observed in the wild-type condition (Fig. 5b, WT $121.7 \pm 7.2 \%$, $n=8$ slices, four mice versus KO $123.7 \pm 4.4 \%, n=7$ slices, three mice; $P=0.72$, Student's $t$-test, values taken from averages of 26$30 \mathrm{~min}$ after induction). Likewise, delivery of an induction protocol with a lesser number of total stimuli, $10 \mathrm{~Hz}$ for $10 \mathrm{~s}$, that was used in previous publications, ${ }^{11}$ resulted in a similar amount of potentiation in WT as well as RIM1 $\alpha-/-$ slices (Fig. $5 \mathrm{c}$, WT $119.2 \pm 2.6, n=7$ cells, five mice versus $\mathrm{KO} 118.6 \pm 7.5, n=8$ cells, five mice, $P=0.94$, Student's $t$-test). 
a

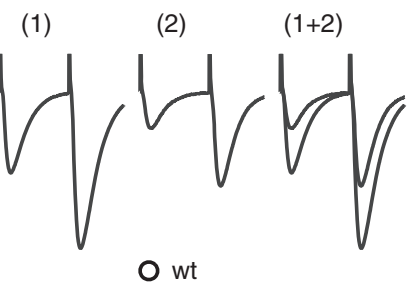

$1 \mathrm{mM} \gamma \mathrm{DGG}$

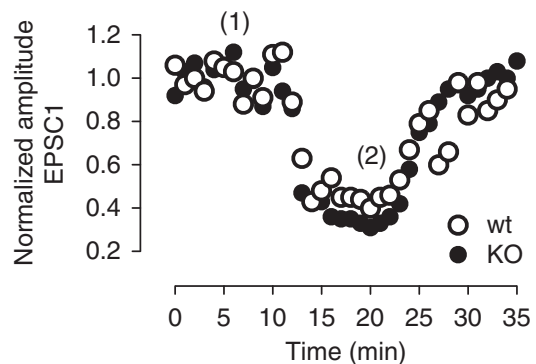

(1)

(2)

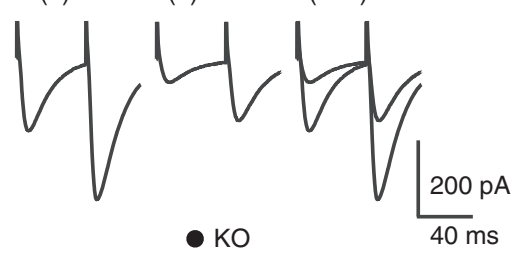

$1 \mathrm{mM} \gamma \mathrm{DGG}$

(1)
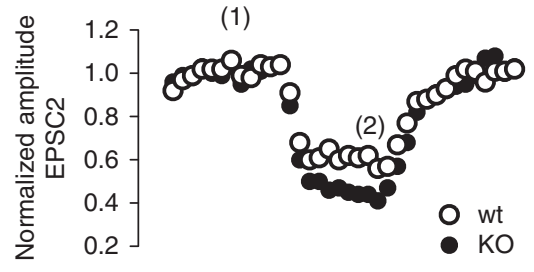

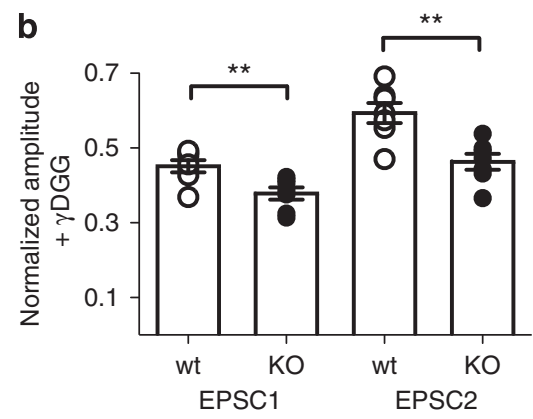

c

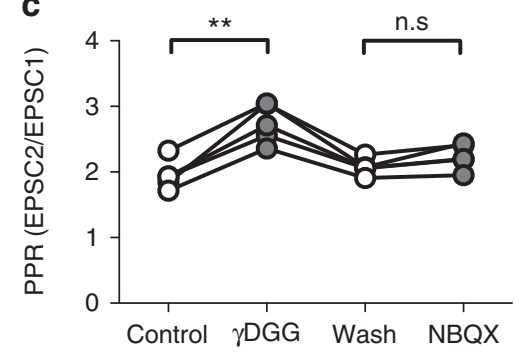

Figure 2 | Decreased synaptic glutamate transients in RIM1 $\boldsymbol{\alpha}$ knockout mice. $1 \mathrm{mM} \gamma$ DGG was applied for 10 min in order to receive relative estimates of the glutamate transient in the synaptic cleft. (a) Normalized amplitude is plotted over time for the first (left) and second (right) pulses of a 50-ms paired pulse. Data points shown are binned to $1 \mathrm{~min}$. Top, example current traces for both genotypes taken at the time points indicated in the graphs below. (b) Summary bar graph for all experiments for the first and second pulses. Data points represent the average of the last 3 min before the washout of $\gamma D G G\left(n=7\right.$ each, bars report mean \pm s.e.m., ${ }^{\star \star} P<0.01$, Student's $t$-test). (c) Summary graph depicting the effects of $\gamma D G G$ and NBQX on the paired-pulse ratio (PPR), measured in the same cells $\left(n=5,{ }^{\star \star} P<0.01\right.$ and n.s. not significant, one-way ANOVA with Bonferroni's multiple comparison).

a

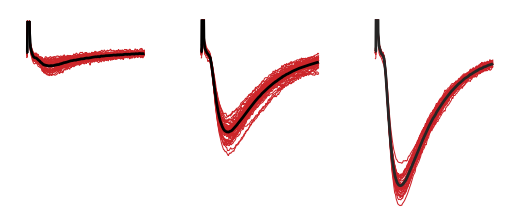

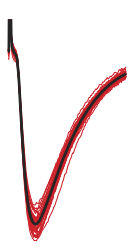
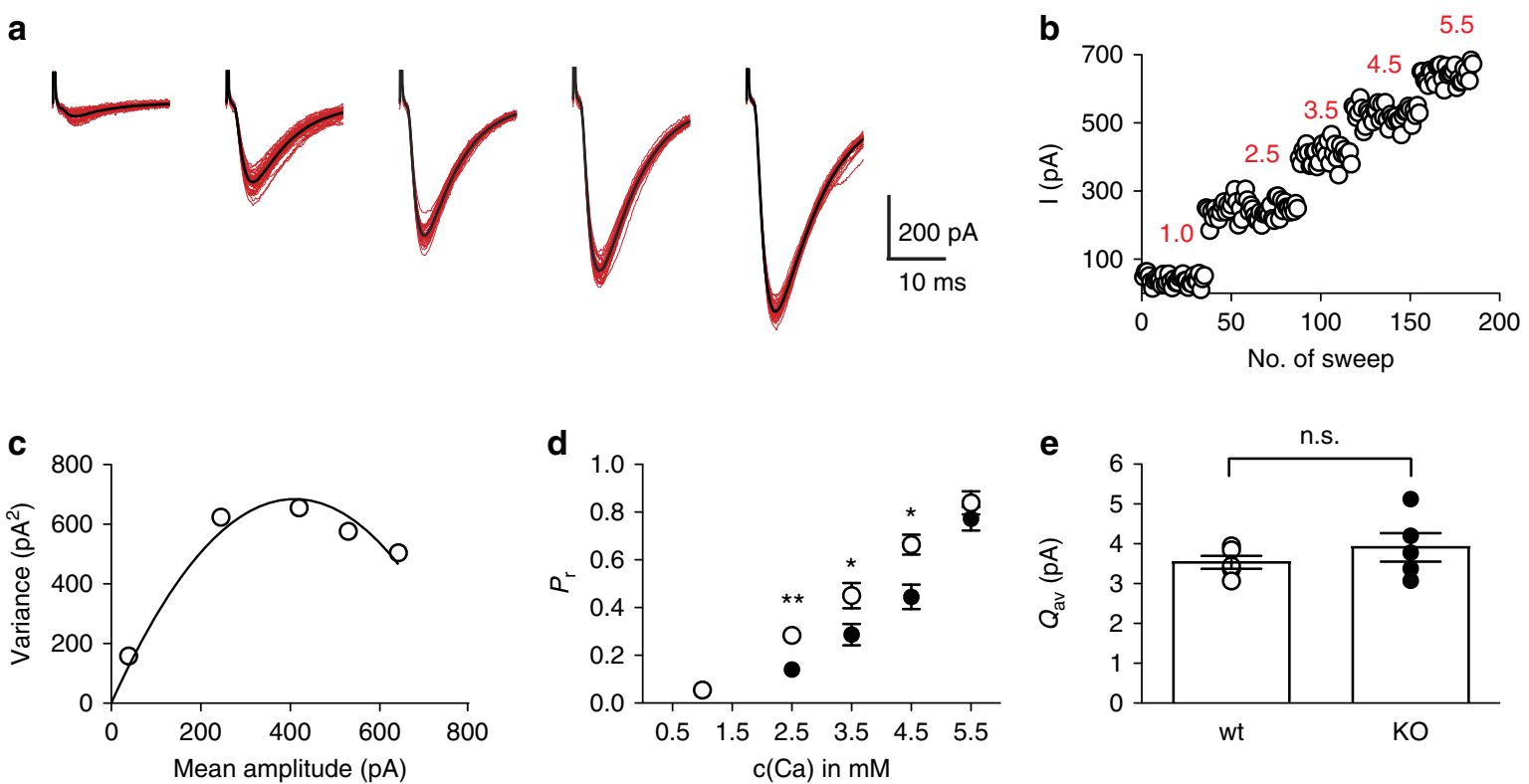

d

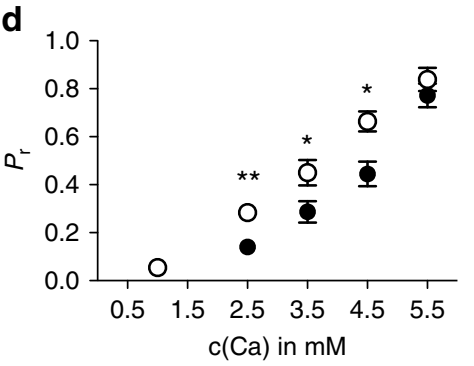

$\mathbf{e}$

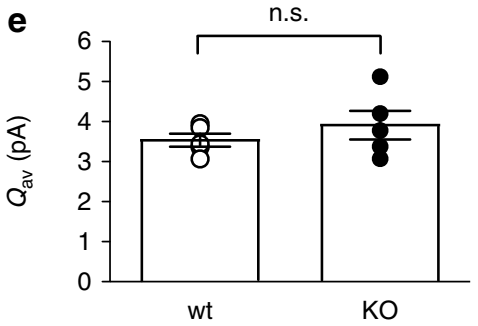

Figure 3 | Reduced release probability in the absence of RIM1 $\alpha$. Variance-mean analysis was performed to quantify differences in the functional synaptic properties of the granule cell-Purkinje cell synapse. (a-c) Representative example from a wild-type slice. (a) EPSCs were recorded under several different $P_{\mathrm{r}}$ conditions by altering $\mathrm{Ca}^{2+}$ to $\mathrm{Mg}^{2}+$ ratio. Single traces (red) and average (black) are shown for (from left to right in $\mathrm{mM}$ ) 1.0, 2.5, 3.5, 4.5 and $5.5 \mathrm{Ca}^{2+}$. (b) Plot of the analysed epochs. Red numbers indicate corresponding $\left[\mathrm{Ca}^{2+}\right]$ in $\mathrm{mM}$. The non-analysed epochs of the wash-in are left out for clarity. (c) Resulting variance-mean plot with respective points (circles) and fitted parabola (black line). Based on these curves, the $P_{\mathrm{r}}$ (d) and averaged quantal content (Q) (e) were calculated for wild type (open circles, $n=5$ ) and RIM1 $\alpha$ KO (filled circles, $n=5$ ); data are reported as mean \pm s.e.m., ${ }^{\star} P<0.05,{ }^{\star \star} P<0.01$ and n.s. not significant, Student's $t$-test.

Of note in this latter set of experiments, there is a substantially stronger post-tetanic potentiation in RIM $1 \alpha-/-$ slices, which can be observed with both tetanic-induction protocols. This fact again most likely reflects the reduced $P_{\mathrm{r}}$ in the RIM1 $\alpha-/-$ as compared with WT. From these data we conclude that the reduction in calcium entry in the absence of RIM $1 \alpha$ does not lead 
a

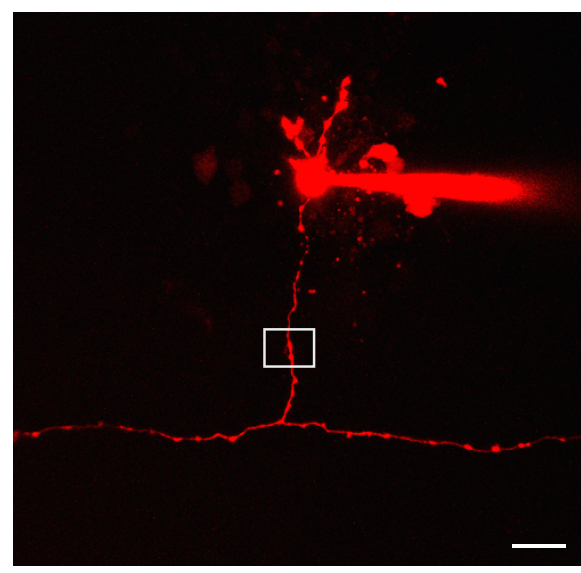

b

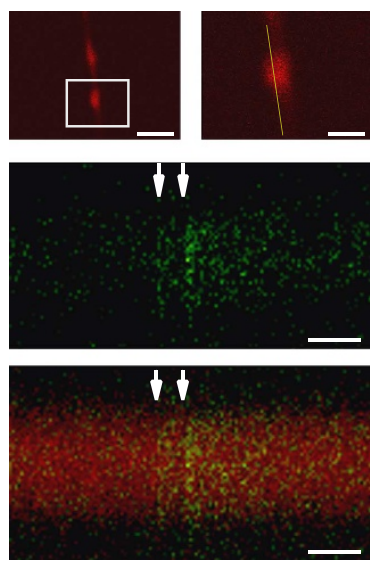

C

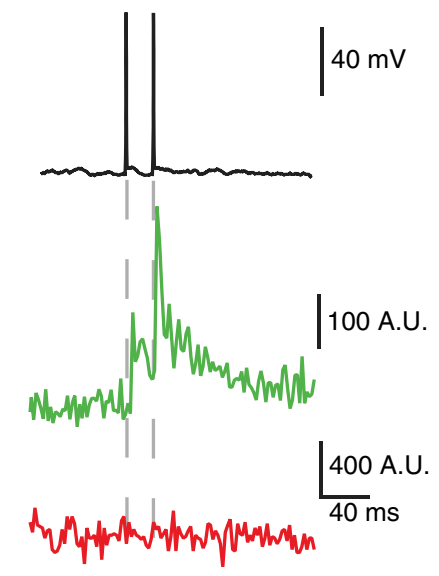

d

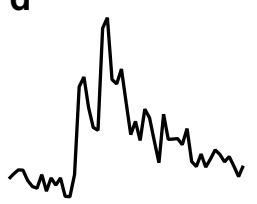

O wt

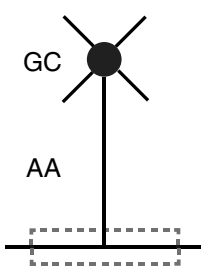

PF

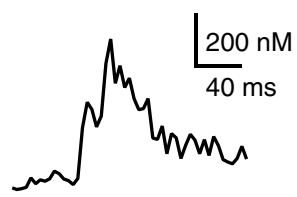

- $\mathrm{KO}$

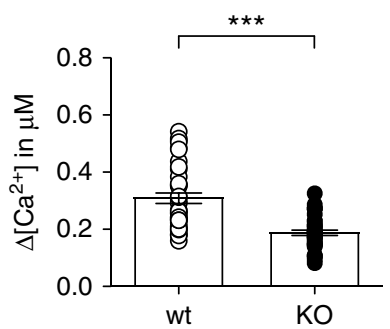

e
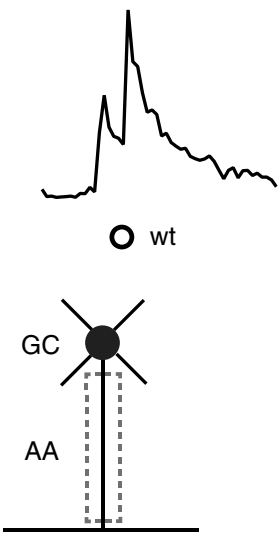

PF
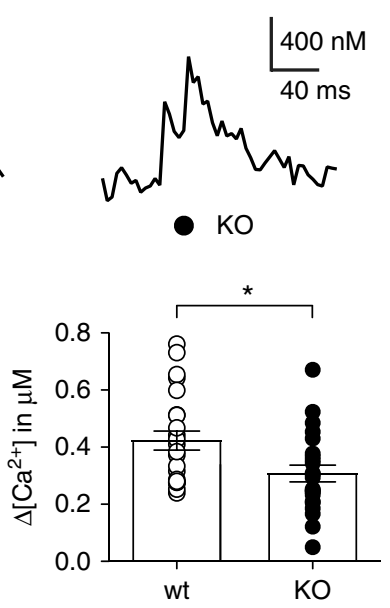

Figure 4 | Reduced presynaptic $\mathbf{C a}^{2}+$ influx in RIM1 $\alpha$-deficient mice. Two-photon laser-scanning fluorescence microscopy was used to assess the Ca ${ }^{2+}$ transient at single boutons of the granule cell ascending axon and the parallel fibre segment. (a) Two-photon image of a cerebellar granule cell filled with Alexa-594 $(30 \mu \mathrm{M})$ and fluo4FF $(200 \mu \mathrm{M})$; scale bar $=20 \mu \mathrm{m}$. (b) Medium (upper left, scale bar $=2 \mu \mathrm{m})$ and high (upper right, scale bar $=1 \mu \mathrm{m}$ ) magnification of a single ascending axon bouton. The yellow line indicates the orientation of the line scan $(500 \mathrm{~Hz})$ for the fluorescence measurement. Two action potentials (white arrows) with an interstimulus interval of $20 \mathrm{~ms}$ were elicited through current injection in the soma and the fluorescence intensity was recorded. The response to the paired action potentials is shown for the green channel (middle, scale bar $=40 \mathrm{~ms}$ ) and the green merged with the red channel (bottom, scale bar $=40 \mathrm{~ms}$ ). (c) Corresponding traces for the single trial shown in (b) of the somatic potential (top), the green channel (middle) and the red channel (bottom). (d) Representative calcium transients (average of four traces, binned to $250 \mathrm{~Hz}$ ) for wild type and knockout and summary graph for both genotypes recorded from parallel fibre (PF) boutons. $\left[\mathrm{Ca}^{2+}\right]$ changes are calculated from the average of four data points (16-ms interval) after the first peak. Bar graph shows average value and data points for individual boutons (open circle corresponds to WT, closed circles to KO);

$\mathrm{n}(\mathrm{WT})=38, \mathrm{n}(\mathrm{KO})=35$, data are reported as mean \pm s.e.m., ${ }^{\star \star \star} P<0.001$. (e) Depicted are the recordings from boutons of the ascending axon with representative traces for each genotype (top) and summary graph (bottom); $n=23$ each, data are mean \pm s.e.m., ${ }^{\star} P<0.05$, Student's $t$-test. Schematics on the left in (d) and (e) illustrate the location of recorded boutons from granule cell (GC) axons.

to a failure of LTP induction and that this protein is not an absolute requirement for the expression of presynaptic LTP at the cerebellar PF synapses.

\section{Discussion}

In this report we describe the functional importance of RIM $1 \alpha$ in setting the $P_{\mathrm{r}}$ at the excitatory cerebellar PF synapse on the Purkinje neuron. We demonstrate that the absence of the single isoform RIM1 $\alpha$ is sufficient to substantially reduce calcium influx in the granule cell axon terminals. Furthermore, as a functional consequence $P_{\mathrm{r}}$ is decreased and STP is enhanced.

In addition, we further show that loss of RIM1 $\alpha$ does neither prevent induction nor expression of LTP, and therefore RIM1 $\alpha$ is not an absolute requirement for the expression of LTP at this synapse.
Two recent publications have demonstrated the role of RIM proteins in the targeting of VDCCs to presynaptic terminals. Loss of all RIM1/2 isoforms led to reductions in calcium current densities $^{18}$ and action potential evoked calcium transients measured with calcium-sensitive dyes ${ }^{19}$. An earlier study in expression systems had shown that RIM can also influence kinetic properties of calcium currents by reducing voltage-dependent inactivation $^{27}$. Our results presented here show a robust reduction in calcium-influx amplitudes in granule cell boutons from mice lacking only the RIM $1 \alpha$ isoform. Notably, we found a $30-40 \%$ calcium-influx reduction in the RIM1 $\alpha \mathrm{KO}$, obviously already accounting for a large proportion of VDCC trafficking or functioning when compared with the $50 \%$ reduction in RIM1/2 KOs. The fact that the calcium-influx reduction in RIM1 $\alpha-/-$ was somewhat smaller than those in the reports of Han et al. ${ }^{18}$ 
a
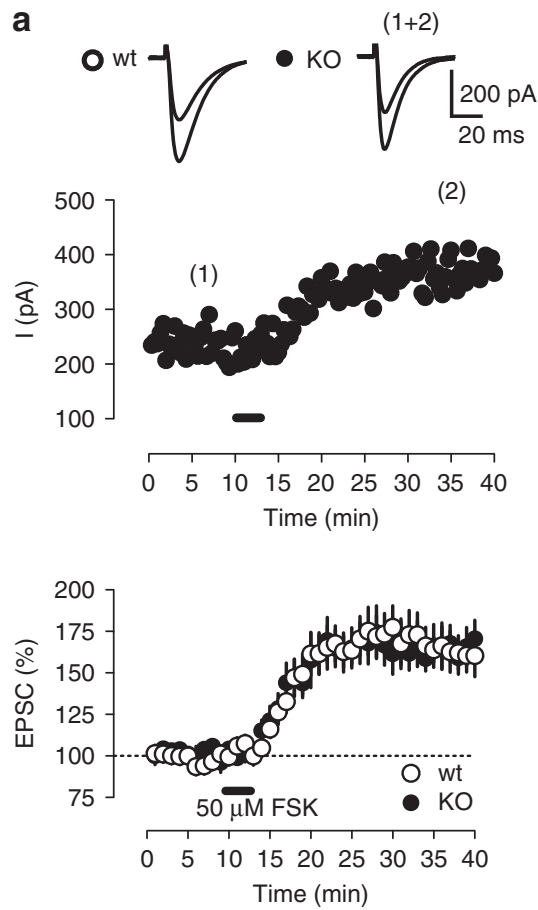

b<smiles>CCC(O)CO</smiles>

- ко $\underbrace{(1+2)}_{\frac{200 \mathrm{pA}}{20 \mathrm{~ms}}}$
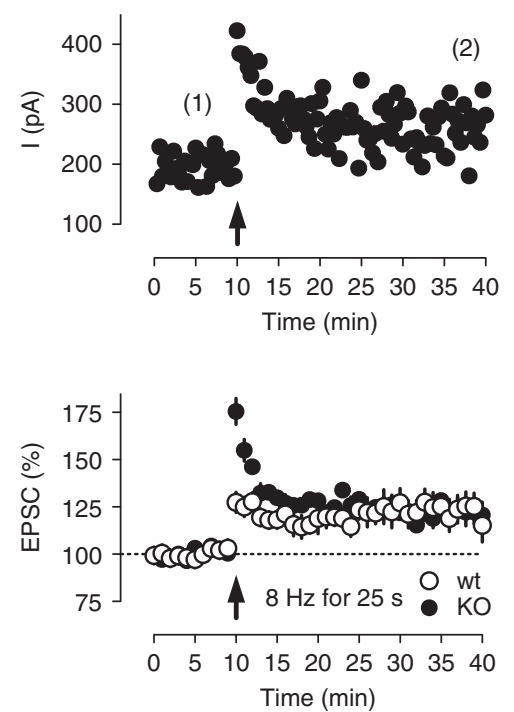

C
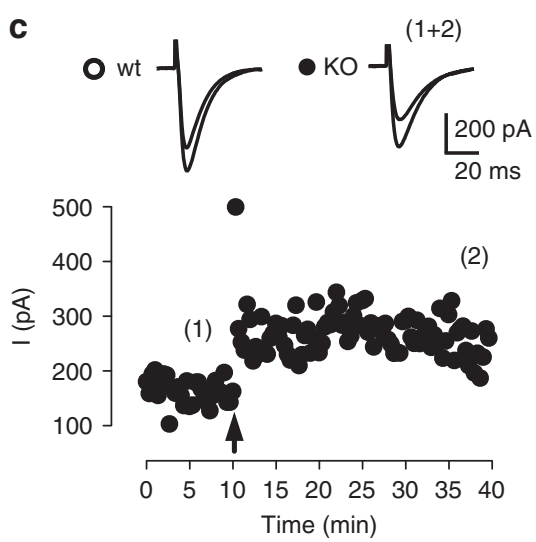

(2)

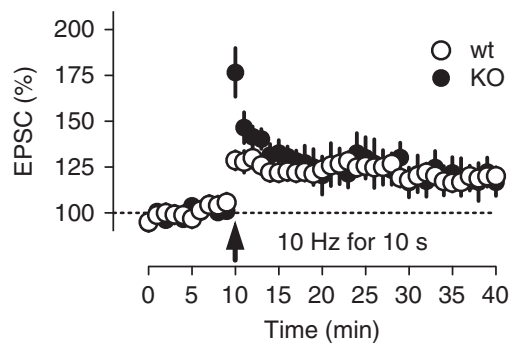

Figure 5 | Presynaptic long-term potentiation is unaltered in RIM1 $\alpha$ knockout mice. LTP was induced by tetanic stimulation or chemically by application forskolin (a-c). (a) Application of forskolin $(50 \mu \mathrm{M})$ for $3 \mathrm{~min}$ (bar); $n=9$ each. (b) Tetanic induction with $8 \mathrm{~Hz}$ for $25 \mathrm{~s}($ arrow); $\mathrm{n}(\mathrm{WT})=8, \mathrm{n}(\mathrm{KO})=7$. (c) Tetanic stimulus protocol consisting of $10 \mathrm{~Hz}$ for $10 \mathrm{~s}$ (arrow); $\mathrm{n}(\mathrm{WT})=7, \mathrm{n}(\mathrm{KO})=8$. In each panel, on top are shown example traces for both genotypes, which represent the average of $5 \mathrm{~min}$ at the time points indicated in the graphs below. Middle shows the time course of a single experiment for RIM1a KO. Bottom displays summary data for WT (open circles) and KO (closed circles), in which values are plotted as mean \pm s.e.m.

and Kaeser et al. ${ }^{19}$ might be explained by the fact that in our study only the RIM1 $\alpha$ isoform was deleted, whereas in the other two studies all RIM1/2 isoforms were knocked out.

The Calyx of Held synapse showed, in addition to reduced calcium currents, a modest decrease in $P_{\mathrm{r}}$ and in turn displayed a reduced depression in response to stimulus trains in the absence of RIM 1 and 2 (ref. 18). The cortico-amygdala synapse is another preparation in which both a reduction in $P_{\mathrm{r}}$ in combination with an increased STP has been described for RIM1 $\alpha \mathrm{KOs}^{17}$. The role of calcium influx was not tested in this latter study. The question remains whether the calcium-influx reduction that we find is responsible for the decrease in apparent $P_{\mathrm{r}}$ ? From our data, assuming that calcium influx through VDCCs is only moderately saturated in the range of 1-5.5 mM external calcium concentration, one can see that a $30 \%$ reduction in external calcium. (3.5$2.5 \mathrm{mM}$ ) leads to some $40 \%$ reduction in $P_{\mathrm{r}}$ in the wild-type situation (see Fig. 3d). This value comes close to the reduction in $P_{\mathrm{r}}$ ranging between 50 and $30 \%$ that we see in $\mathrm{KO}$ mice (over calcium concentrations from 2.5 to $4.5 \mathrm{mM}$ ), in which we found a decrease in calcium influx of roughly $30 \%$ in comparison with wild type. In addition, the deficit in $P_{\mathrm{r}}$ in RIM1 $\alpha$ KOs can be overcome by raising extracellular calcium concentration to higher levels (that is, $5.5 \mathrm{mM}$ ), where $P_{\mathrm{r}}$ values reach the same magnitude as in the wild-type condition. Moreover, we also found smaller calcium transients in boutons from the PF segment of granule cells in comparison with boutons from the ascending axon segment (Fig. 4d,e). This observation corresponds well with a previous study by Sims and Hartell ${ }^{25}$ demonstrating a smaller $P_{\mathrm{r}}$ at synapses from the PF as compared with the ascending axon, further emphasizing the importance of calcium influx for $P_{\mathrm{r}}$. Therefore, our data indicate that calcium-influx reduction is a crucial factor for the reduced $P_{\mathrm{r}}$ at the parallel fibre to Purkinje cell synapse.
Furthermore, it is also very well conceivable that reductions in calcium influx may even lead to a complete shutdown of functional release sites $(\mathrm{N})$ in RIM1 $\alpha \mathrm{KO}$ mice. In our variancemean analysis approach, the number of release sites are primarily dependent on the amount of activated fibres, which is in turn set by the stimulation strength and position. As this cannot be adjusted with the desired precision between slices and genotypes, we had to refrain from calculating the parameter N. However, it remains an intriguing thought that the functional number of release sites could be reduced in RIM1 $\alpha$-deficient mice, and at the current stage we cannot exclude the possibility that the reduction in apparent $P_{\mathrm{r}}$ may in part stem from a reduced number of functional release sites $(\mathrm{N})$.

However, VDCC targeting is seemingly not the only effect of RIMs that may result in reduced $P_{\mathrm{r}}$, as both the number of docked vesicles as well as the calcium sensitivity for transmitter release can be also affected by RIM deletion ${ }^{18}$. For this reason, further functional properties besides interaction with VDCCs of RIM proteins have also to be taken into consideration, which can additionally affect the reduction in $P_{\mathrm{r}}$ besides reduced calcium influx. Those parameters could not be tested in the present study. Very likely it is a combination of all those factors acting in concert to set the $P_{r}$; however, RIM1 $\alpha$ 's influence on VDCCs already seems to have a major impact on $P_{\mathrm{r}}$ at the cerebellar parallel fibre synapse.

In the majority of synapses tested so far, disruption of essential components of the priming or release apparatus has led to profound changes in the synapse STP behaviour (mostly meaning an increase in short-term facilitation), if transmission was not completely abolished. Such results have been described, for example, for complexins ${ }^{28,29}$, SV2 (refs 30,31), the RIM interaction partners Munc13 $\mathrm{s}^{32,33}$ and Rab3a ${ }^{34}$, as well as RIM1 $\alpha$ itself $^{13}$. In most of the reported cases, the STP changes 
were accompanied by or caused by a reduction in $P_{\mathrm{r}}$. A notable exception in this context is Rab3A, in which increased short-term facilitation was described with no alteration in $P_{\mathrm{r}}$ at Schaffercollateral synapses ${ }^{13,34}$. Only the complete deletion of all four Rab3 isoforms A-D was found to lead to reduced $P_{\mathrm{r}}^{15,35}$. In the case of RIM1 $\alpha$ and Rab3a, the situation was particularly special as their loss led to STP changes only in a synapse-specific manner: Increased short-term facilitation at the Schaffer collateral synapses ${ }^{13}$ but unchanged STP at the hippocampal mossy fibre and cerebellar PF connections (in those latter cases they were essential for long-term potentiation instead $)^{11}$. Our data instead add the PF synapse to the group of connections in which RIM1 $\alpha$ deletion results in alterations of STP, which is explained by the described calcium-influx reduction.

The currently held view on the mechanism of presynaptically expressed forms of LTP holds that RIM1 $\alpha$ is an essential mediator in the expression of this process $5,22,36,37$. Two groups have found that in different preparations loss of RIM1 $\alpha$ abolishes the expression of LTP at connections such as hippocampal mossy fibres, ${ }^{11}$ cerebellar parallel fibres ${ }^{11}$ and cortico-amygdala synapses ${ }^{17}$.

Considering the recent data demonstrating the interaction of RIM1 $\alpha$ with VDCCs and therefore presynaptic terminal calcium currents $^{18,19}$, one could have speculated that the absence of LTP might be due to a failure of induction rather than expression because of insufficient calcium entry (even though this was taken into account in the original description of the LTP deficiency by raising extracellular calcium concentrations during induction, which was still not capable of rescuing $\mathrm{LTP}^{11}$ ). However, in contrast we are able to show in this study that despite the absence of RIM1 $\alpha$, LTP can be induced normally at cerebellar PF synapses. These data challenge the view of RIM1 $\alpha$ being an essential mediator of presynaptic LTP expression, but rather point to a role in altering the threshold for induction.

The fact that PF-LTP can be readily obtained in conditions of reduced calcium entry is in line with other data in the literature. Pharmacologically blocking VDCCs responsible for presynaptic calcium entry such as $\mathrm{P} / \mathrm{Q}-, \mathrm{N}$-type channels or reducing extracellular calcium concentration that reduce calcium influx to $20-50 \%$ did also not prevent the establishment of LTP at hippocampal mossy fibres ${ }^{38}$ or cerebellar parallel fibres ${ }^{39}$.

One possible explanation for the apparent discrepancy of our data with previously published results might be that a very subtle threshold level for LTP induction exists. This threshold could be sensitive to slight alterations in the level of extra- or intracellular calcium concentration or resting membrane potentials. The study of Myoga and Regehr ${ }^{39}$, for example, demonstrated that a small reduction of extracellular calcium from 1.5 to just $1.25 \mathrm{mM}$ prevented the induction of LTP. In line with this latter finding we also found a loss of LTP in $1.25 \mathrm{mM}$ external calcium, which is indicative of a rather sharp threshold for LTP induction. As for the chemical LTP induction with forskolin or cAMP analogues, there were already conflicting views in the literature whether or not in this situation RIM1 $\alpha$ was necessary ${ }^{11,40}$. Moreover, mutation of a putative rotein kinase A (PKA) phosphorylation site on RIM1 $\alpha$ was also found to not prevent presynaptic LTP ${ }^{41}$, casting additional doubt on the function of RIM1 $\alpha$ as the ultimate target for presynaptic LTP expression.

Whatever the underlying reason for the different results may be, our study might motivate further explorations into alternative molecular mechanisms of the expression of presynaptic LTP. Our results are more in line with a general and broad non-synapsespecific role of RIM in excitatory connections, in which its interaction with VDCCs ensures proper release and STP characteristics of synapses.

\section{Methods}

Preparation of acute cerebellar tissue slices. All experiments were carried out in accordance with the local and national guidelines (Berlin State Government, T0100/03).

RIM1 $\alpha$ KO mice and wild-type littermates of both sexes (3-6 weeks) were anesthetized and decapitated. The brain was quickly removed and cooled down in either low $\left[\mathrm{Ca}^{2+}\right]$ or (for $\mathrm{Ca}^{2+}$-imaging experiments) sucrose-based ACSF containing (in mM): $119 \mathrm{NaCl}, 26 \mathrm{NaHCO}_{3}, 10$ glucose, $2.5 \mathrm{KCl}, 1 \mathrm{NaH}_{2} \mathrm{PO}_{4}, 0.5$ $\mathrm{CaCl}_{2}$ and $1.3 \mathrm{MgCl}_{2}$ or $87 \mathrm{NaCl}, 26 \mathrm{NaHCO}_{3}, 50$ sucrose, 10 glucose, $2.5 \mathrm{KCl}, 1.25$ $\mathrm{NaH}_{2} \mathrm{PO}_{4}, 0.5 \mathrm{CaCl}_{2}$ and $3 \mathrm{MgCl}_{2}$. Solutions were constantly oxygenated with carbogen $\left(5 \% \mathrm{O}_{2}\right.$ and $\left.95 \% \mathrm{CO}_{2}\right)$.

Cerebellar slices $(300 \mu \mathrm{m}$, sagittal or horizontal) were cut with a Leica VT1200S microtome (Wetzlar, Germany) and stored in the above solutions for $30 \mathrm{~min}$ at $35^{\circ} \mathrm{C}$. Subsequently, slices were stored in recording solution containing the following (in mM): $119 \mathrm{NaCl}, 26 \mathrm{NaHCO}_{3}, 10$ glucose, $2.5 \mathrm{KCl}, 1 \mathrm{NaH}_{2} \mathrm{PO}_{4}, 2.5$ $\mathrm{CaCl}_{2}$ and $1.3 \mathrm{MgCl}_{2}$ at room temperature, and experiments were started after $30 \mathrm{~min}$.

Electrophysiological recordings. Whole-cell recordings of Purkinje cells were carried out in the sagittal slices with a MultiClamp 700B (Axon Instruments, Union City, CA, USA) amplifier; signals were filtered at $2 \mathrm{kHz}$ and digitized (National Instruments, BNC-2090) at $5 \mathrm{kHz}$, and recorded and analysed with custom-made software in IGOR Pro (WaveMetrics Inc., OR, USA). Experiments were performed in the presence of the $\mathrm{GABA}_{\mathrm{A}}$ receptor antagonist SR 95531 hydrobromide $(1 \mu \mathrm{M})$. For recording borosilicate glass electrodes $(2-5 \mathrm{M} \Omega)$ were filled with intracellular solution containing the following (in $\mathrm{mM}$ ): $135 \mathrm{~K}$-Gluconate, $20 \mathrm{KCl}, 2 \mathrm{MgATP}, 10$ HEPES, 0.5 EGTA and 5 phosphocreatine. For extracellular fibre stimulation, a low-resistance patch pipette was filled with ACSF and placed in the molecular layer of the cerebellum, almost perpendicular to the Purkinje cell layer and the patched neuron. Stimulation frequency was $0.05 \mathrm{~Hz}$ or is mentioned in the text if otherwise. The tetanic-induction protocol for LTP consisted either of a $8-\mathrm{Hz}$ train applied once for $25 \mathrm{~s}$ (Fig. 5b) or a 10-Hz train applied for $10 \mathrm{~s}$ (Fig. 5c). Chemical LTP was induced by applying forskolin at a concentration of $50 \mu \mathrm{M}$ for $3 \mathrm{~min}$. Recordings were carried out at room temperature. Series and input resistances $\left(\mathrm{R}_{\mathrm{s}}, \mathrm{R}_{\mathrm{i}}\right)$ were constantly monitored, and experiments were discarded if changes in the $\mathrm{R}_{\mathrm{s}}$ were $>15 \%$

Two-photon calcium imaging in granule cell boutons. Two-photon $\mathrm{Ca}^{2+}$ imaging was performed in horizontal slices at $34^{\circ} \mathrm{C}$. Granule cells were patched with thick-walled borosilicate glass electrodes (6-10 $\mathrm{M} \Omega$ ) filled with the following (in $\mathrm{mM}$ ): $114 \mathrm{KMeSO}_{4}, 10 \mathrm{HEPES}, 4 \mathrm{MgATP}, 0.4 \mathrm{NaGTP}$ and 14 phosphocreatine. The solution was complemented with $30 \mu \mathrm{M}$ Alexa-594 for neuronal tracing and $200 \mu \mathrm{M}$ Fluo4FF as intracellular $\mathrm{Ca}^{2}+$ indicator. Pairs of action potentials for opening of VDCCs were evoked through current injection (250-600 pA) for $1 \mathrm{~ms}$ with an interstimulus interval of $20 \mathrm{~ms}$.

Experiments were started after 20 min of loading the cell with Alexa-594 and Fluo4FF to ensure a proper diffusion of the dyes and stable concentrations at the site of interest. Fluometric $\mathrm{Ca}^{2}+$ measurements were performed with a twophoton laser-scanning system (Femto2D; Femtonics, Hungary) equipped with a femtosecond laser tuned to 805 or $810 \mathrm{~nm}$ (Chameleon; Coherent). Epifluorescence was collected with an Olympus LUMPlanFL N $(60 \times / 1.0 \mathrm{NA})$ water-immersion objective, and for transfluorescence an Olympus oil-immersion condenser (1.4 NA) was used. Fluorescence was divided by a dichroic mirror at $\sim 590-600 \mathrm{~nm}$, and green and red signals were filtered using 525/50 and 650/50 bandpass filters, respectively. A diagram of the light path can be obtained from Chiovini et al. ${ }^{42}$ Measurement control, data acquisition and analysis were performed using the Matlab-based MES program package (Femtonics). Line scans for fluometric measurements were performed with $500 \mathrm{~Hz}$. In order to calculate $\Delta\left[\mathrm{Ca}^{2+}\right]$ from the corresponding $\Delta G / R$ values, $(G / R)_{\min }$ and $(G / R)_{\max }$ were determined under conditions of $0\left[\mathrm{Ca}^{2+}\right]$ and saturating $\left[\mathrm{Ca}^{2+}\right]$ by adding $2 \mathrm{mM}$ EGTA or $2 \mathrm{mM}$ $\mathrm{CaCl}_{2}$ to the pipette 23,43 . $(G / R)_{\max }$ values under physiological conditions were validated by application of a $500-\mathrm{Hz}$ train of APs for $1 \mathrm{~s}$ at the end of some experiments and similar $(G / R)_{\max }$ ratios could be obtained. The procedure was repeated for all calcium-indicator stocks and wavelengths used. $\left[\mathrm{Ca}^{2+}\right]$ was then calculated according to the following equation with a $K_{\mathrm{d}}=8.1 \mu \mathrm{M}^{23}$ :

$$
\frac{\left[\mathrm{Ca}^{2+}\right]}{K_{\mathrm{d}}}=\frac{\left(\frac{G}{R}\right)-\left(\frac{G}{R}\right)_{\text {min }}}{\left(\frac{G}{R}\right)_{\text {max }}-\left(\frac{G}{R}\right)_{\text {min }}}
$$

Data analysis and statistics. To construct the variance-mean plot, EPSCs were recorded at five different release probabilities by altering $\mathrm{Ca}^{2}+/ \mathrm{Mg}^{2}+$ ratio. For every condition, epochs of at least 30 sweeps were chosen and controlled by a Spearman rank correlation test to fit the stability criterion. After subtraction of baseline noise variance, a simple parabola was fitted to the resulting variance-mean plot, which in all cases passed through a maximum. Experiments were excluded if the goodness of fit was $R^{2}<0.7$. Weighted basal synaptic parameters $\left(P_{\mathrm{r}}, Q_{\mathrm{av}}\right)$ were determined $^{44,45}$ according to the following equation:

$$
y=A \times \bar{x}-B \times \bar{x}^{2}
$$


with

$$
Q_{\mathrm{av}}=\frac{A}{1+C V^{2}}
$$

and

$$
\mathrm{P}_{\mathrm{r}}=\bar{x} \frac{B}{A}\left(1+C V^{2}\right)
$$

$y=$ variance; $\bar{x}=$ mean amplitude; $C V$ (Coefficient of variation of EPSC at individual release sites) $=0.3$.

Statistical data analysis was performed using GraphPad Prism 5 (Graph Pad Software, La Jolla, USA). Results were considered significant if $P<0.05$ ( $^{*}$ and ${ }^{* *}$ for $P<0.01$ ) using an unpaired Student's $t$-test, except for Fig. $2 c$, where a one-way ANOVA with Bonferroni's multiple comparison was used. All numerical values are given as mean \pm s.e.m.

Drugs and chemical compounds. SR 95531 hydrobromide and $\gamma$ DGG were purchased from Tocris/Biozol (Eching, Germany), Alexa-594 and Fluo4FF from Invitrogen/Life Technologies (Darmstadt, Germany) and ACSF compounds from Roth (Karlsruhe, Germany).

\section{References}

1. Fioravante, D. \& Regehr, W. G. Short-term forms of presynaptic plasticity Curr. Opin. Neurobiol. 21, 269-274 (2011).

2. Zucker, R. S. \& Regehr, W. G. Short-term synaptic plasticity. Annu. Rev. Physiol. 64, 355-405 (2002).

3. Atwood, H. L. \& Karunanithi, S. Diversification of synaptic strength: presynaptic elements. Nat. Rev. Neurosci. 3, 497-516 (2002).

4. Malenka, R. C. \& Nicoll, R. A. Long-term potentiation--a decade of progress? Science 285, 1870-1874 (1999).

5. Malenka, R. C. \& Bear, M. F. LTP and LTD: an embarrassment of riches. Neuron 44, 5-21 (2004).

6. Zalutsky, R. A. \& Nicoll, R. A. Comparison of two forms of long-term potentiation in single hippocampal neurons. Science 248, 1619-1624 (1990).

7. Salin, P. A., Malenka, R. C. \& Nicoll, R. A. Cyclic AMP mediates a presynaptic form of LTP at cerebellar parallel fibre synapses. Neuron 16, 797-803 (1996).

8. Humeau, Y., Shaban, H., Bissiere, S. \& Luthi, A. Presynaptic induction of heterosynaptic associative plasticity in the mammalian brain. Nature 426, 841-845 (2003).

9. Wozny, C., Maier, N., Schmitz, D. \& Behr, J. Two different forms of long-term potentiation at CA1-subiculum synapses. J. Physiol. 586, 2725-2734 (2008).

10. Dulubova, I. et al. A Munc13/RIM/Rab3 tripartite complex: from priming to plasticity? EMBO. J. 24, 2839-2850 (2005)

11. Castillo, P. E., Schoch, S., Schmitz, F., Sudhof, T. C. \& Malenka, R. C. RIM1alpha is required for presynaptic long-term potentiation. Nature 415, 327-330 (2002).

12. Chevaleyre, V., Heifets, B. D., Kaeser, P. S., Sudhof, T. C. \& Castillo, P. E. Endocannabinoid-mediated long-term plasticity requires cAMP/PKA signaling and RIMlalpha. Neuron 54, 801-812 (2007).

13. Schoch, S. et al. RIMlalpha forms a protein scaffold for regulating neurotransmitter release at the active zone. Nature 415, 321-326 (2002).

14. Geppert, M. et al. The role of Rab3A in neurotransmitter release. Nature 369, 493-497 (1994).

15. Schluter, O. M., Basu, J., Sudhof, T. C. \& Rosenmund, C. Rab3 superprimes synaptic vesicles for release: implications for short-term synaptic plasticity. J. Neurosci. 26, 1239-1246 (2006).

16. Castillo, P. E. et al. Rab3A is essential for mossy fibre long-term potentiation in the hippocampus. Nature 388, 590-593 (1997).

17. Fourcaudot, E. et al. cAMP/PKA signaling and RIMlalpha mediate presynaptic LTP in the lateral amygdala. Proc. Natl. Acad. Sci. USA 105, 15130-15135 (2008).

18. Han, Y., Kaeser, P. S., Sudhof, T. C. \& Schneggenburger, R. RIM determines $\mathrm{Ca}(2)+$ channel density and vesicle docking at the presynaptic active zone. Neuron 69, 304-316 (2011)

19. Kaeser, P. S. et al. RIM proteins tether $\mathrm{Ca} 2+$ channels to presynaptic active zones via a direct PDZ-domain interaction. Cell 144, 282-295 (2011).

20. Deng, L., Kaeser, P. S., Xu, W. \& Sudhof, T. C. RIM proteins activate vesicle priming by reversing autoinhibitory homodimerization of Munc13. Neuron 69, 317-331 (2011)

21. Nicoll, R. A. \& Schmitz, D. Synaptic plasticity at hippocampal mossy fibre synapses. Nat. Rev. Neurosci. 6, 863-876 (2005).

22. Castillo, P. E. Presynaptic LTP and LTD of excitatory and inhibitory synapses. Cold Spring Harb. Perspect. Biol. 4, a005728-a005728 (2012).

23. Yasuda, R. et al. Imaging calcium concentration dynamics in small neuronal compartments. Sci. STKE. 2004, 15 (2004).

24. Brenowitz, S. D. \& Regehr, W. G. Reliability and heterogeneity of calcium signaling at single presynaptic boutons of cerebellar granule cells. J. Neurosci. 27, 7888-7898 (2007).
25. Sims, R. E. \& Hartell, N. A. Differences in transmission properties and susceptibility to long-term depression reveal functional specialization of ascending axon and parallel fibre synapses to Purkinje cells. J. Neurosci. 25, 3246-3257 (2005).

26. Sims, R. E. \& Hartell, N. A. Differential susceptibility to synaptic plasticity reveals a functional specialization of ascending axon and parallel fibre synapses to cerebellar Purkinje cells. J. Neurosci. 26, 5153-5159 (2006).

27. Kiyonaka, S. et al. RIM1 confers sustained activity and neurotransmitter vesicle anchoring to presynaptic Ca2 + channels. Nat. Neurosci. 10, 691-701 (2007).

28. Xue, M. et al. Complexins facilitate neurotransmitter release at excitatory and inhibitory synapses in mammalian central nervous system. Proc. Natl. Acad. Sci. USA 105, 7875-7880 (2008).

29. Reim, K. et al. Complexins regulate a late step in $\mathrm{Ca} 2+-$ dependent neurotransmitter release. Cell 104, 71-81 (2001).

30. Custer, K. L., Austin, N. S., Sullivan, J. M. \& Bajjalieh, S. M. Synaptic vesicle protein 2 enhances release probability at quiescent synapses. J. Neurosci. 26, 1303-1313 (2006).

31. Chang, W. P. \& Sudhof, T. C. SV2 renders primed synaptic vesicles competent for Ca2 + -induced exocytosis. J. Neurosci. 29, 883-897 (2009).

32. Rosenmund, C. et al. Differential control of vesicle priming and short-term plasticity by Munc13 isoforms. Neuron 33, 411-424 (2002).

33. Breustedt, J. et al. Munc13-2 differentially affects hippocampal synaptic transmission and plasticity. Cereb. Cortex 20, 1109-1120 (2010).

34. Geppert, M., Goda, Y., Stevens, C. F. \& Sudhof, T. C. The small GTP-binding protein Rab3A regulates a late step in synaptic vesicle fusion. Nature 387, 810-814 (1997).

35. Schluter, O. M., Schmitz, F., Jahn, R., Rosenmund, C. \& Sudhof, T. C. A complete genetic analysis of neuronal Rab3 function. J. Neurosci. 24, 6629-6637 (2004).

36. Kaeser, P. S. \& Sudhof, T. C. RIM function in short- and long-term synaptic plasticity. Biochem. Soc. Trans. 33, 1345-1349 (2005).

37. Ho, V. M., Lee, J. A. \& Martin, K. C. The cell biology of synaptic plasticity Science 334, 623-628 (2011).

38. Castillo, P. E., Weisskopf, M. G. \& Nicoll, R. A. The role of Ca2 + channels in hippocampal mossy fibre synaptic transmission and long-term potentiation. Neuron 12, 261-269 (1994).

39. Myoga, M. H. \& Regehr, W. G. Calcium microdomains near R-type calcium channels control the induction of presynaptic long-term potentiation at parallel fibre to purkinje cell synapses. J. Neurosci. 31, 5235-5243 (2011).

40. Lonart, G. et al. Phosphorylation of RIMlalpha by PKA triggers presynaptic long-term potentiation at cerebellar parallel fibre synapses. Cell 115, 49-60 (2003).

41. Kaeser, P.S. et al. RIM1alpha phosphorylation at serine- 413 by protein kinase A is not required for presynaptic long-term plasticity or learning. Proc. Natl. Acad. Sci. USA 105, 14680-14685 (2008).

42. Chiovini, B. et al. Enhanced dendritic action potential backpropagation in parvalbumin-positive basket cells during sharp wave activity. Neurochem. Res 35, 2086-2095 (2010).

43. Grynkiewicz, G., Poenie, M. \& Tsien, R. Y. A new generation of $\mathrm{Ca} 2+$ indicators with greatly improved fluorescence properties. J. Biol. Chem. 260, 3440-3450 (1985).

44. Reid, C. A. \& Clements, J. D. Postsynaptic expression of long-term potentiation in the rat dentate gyrus demonstrated by variance-mean analysis. J. Physiol. 518(Pt 1): 121-130 (1999).

45. Clements, J. D. \& Silver, R. A. Unveiling synaptic plasticity: a new graphical and analytical approach. Trends Neurosci. 23, 105-113 (2000).

\section{Acknowledgements}

We would like to thank S. Rieckmann for excellent technical assistance. The study was supported by grants from the DFG (Exc 257 and SFB 618) and by the BMBF (BCCN grant number 01GQ0410).

\section{Author contributions}

M.K., C.W., F.J., D.S. and J.B. designed the research; M.K. performed the research; M.K. and J.B. analysed data; M.K., D.S. and J.B. wrote the paper.

\section{Additional information}

Competing financial interests: The authors declare no competing financial interests.

Reprints and permission information is available online at http://npg.nature.com/ reprintsandpermissions/

How to cite this article: Kintscher, M. et al. Role of RIM1 $\alpha$ in short- and long-term synaptic plasticity at cerebellar parallel fibres. Nat. Commun. 4:2392 doi: 10.1038/ ncomms3392 (2013). 